ISSN 0103-9954

\title{
COMPOSIÇÃO E ESTRUTURA DO COMPONENTE ARBÓREO DE MATA COM ACURI NO PANTANAL MATOGROSSENSE, BRASIL
}

\author{
TREE SPECIES COMPOSITION AND STRUCTURE IN AN AREA REPRESENTATIVE OF “ACURI \\ FOREST” AT PANTANAL MATOGROSSENSE, BRASIL
}

\author{
Raquel Rejane Bonato Negrelle ${ }^{1}$
}

\begin{abstract}
RESUMO
Visando contribuir para o maior conhecimento deste tipo florestal pantaneiro, apresenta-se resultado de levantamento florístico-estrutural realizado em área vulgarmente denominada de "Mata com Acuri", localizada na RPPN SESC Pantanal (Município de Barão de Melgaço - MT, Brasil - 16 $6^{\circ} 45^{\prime}$ S; 56 $6^{\circ} 15^{\prime}$ ' W). Os dados florísticos foram obtidos a partir de coletas sistemáticas de material botânico fértil e vegetativo efetuadas na área amostral e entorno desta. Para coleta de dados estruturais, a área foi subdividida em 100 parcelas contíguas de $10 \mathrm{~m} \times 10 \mathrm{~m}$. Dentro de cada uma destas parcelas, todos os indivíduos arbóreos com diâmetro a altura do peito igual ou superior a $5,0 \mathrm{~cm}$ e palmeiras foram identificados, mensurados e mapeados, utilizando-se o sistema de coordenadas. Foram amostrados 1224 indivíduos ha ${ }^{-1}$, representativos de 44 espécies, 43 gêneros e 27 famílias; índice de diversidade $H^{\prime}=1,613$ (var. 0,003514). Famílias com maior diversidade: Fabaceae (9 spp.), Sapindaceae e Anacardiaceae (3 spp. cada), Bignoniaceae, Malvaceae, Moraceae e Rubiaceae ( $2 \mathrm{spp}$. cada). Dentre as espécies amostradas, 39\% eram perenifólias; $32 \%$ semidecíduas e $29 \%$ decíduas. Do total de indivíduos arbóreos amostrados $(\mathrm{n}=440), 51 \%$ eram decíduos; $23 \%$ semidecíduos e $26 \%$ perenifólios. Attalea phalerata foi a espécie que com maior valor de importância $(\mathrm{VI}=121,62)$, devido à sua alta densidade ( $64 \%$ do total de indivíduos amostrados), frequência $(100 \%)$ e elevadíssima área basal $\left(74,10 \mathrm{~m}^{2} . \mathrm{ha}^{-1}\right)$. Com valores subsequentes registraram-se Anadenanthera colubrina, Triplaris americana e Phyllostylon rhamnoide (VI $=35,41 ; 28,51 ; 18,5$, respectivamente). A área estudada foi identificada como bastante peculiar em relação a outros locais representativos de Floresta Estacional, especialmente pela presença massiva de Attalea phalerata e ausência de representantes de Myrtaceae. A grande representatividade de espécies pioneiras em associação à elevada densidade de indivíduos dentro desta categoria sucessional reforça o caráter de formação transicional da Mata com Acuri. Em função da alta porcentagem de espécies decíduas e semidecíduas registradas para a área de estudo, classificou-se esta como sendo representativa de Floresta Estacional Decidual.
\end{abstract}

Palavras-chave: Floresta Estacional Decidual Aluvial; florística; fitossociologia.

\begin{abstract}
Aiming to contribute to the better understanding of this kind of Pantanal forest, the results from a floristicstructural survey performed in a 1ha plot commonly classified as "Acuri Forest", located at RPPN SESC Pantanal (Municipality of Barão de Melgaço - MT, Brazil - $16^{\circ} 45^{\prime}$ 'S; $56^{\circ} 15^{\prime}$ W) are presented. The floristic data were obtained from systematic collection of botanical material on the studied area and surroundings. The structural data were obtained from a one ha plot, divided into 100 contiguous sub-plots $(10 \mathrm{~m} \times 10 \mathrm{~m})$. Inside these sub-plots, all arboreal individuals with diameter at breast high equal or greater than $5.0 \mathrm{~cm}$, including palms, were identified, measured and mapped, using a coordinate system. 1224 individual ha ${ }^{-1}$ were sampled, representing 44 species, 43genus and 27 families; diversity index $H^{\prime}=1.613$ (var. 0.003514). The highest diversity families were Fabaceae (9 spp.), Sapindaceae e Anacardiaceae (3 spp. each), Bignoniaceae, Malvaceae, Moraceae e Rubiaceae (2 spp. each). Among the sampled species, $39 \%$ were evergreen; $32 \%$ semideciduous and $29 \%$ deciduous. Considering the tree individuals total $(\mathrm{n}=440), 51 \%$

1 Bióloga, Dr., Professora Associada do Departamento de Botânica, Universidade Federal do Paraná, Caixa Postal 19031, CEP 81531-990, Curitiba (PR), Brasil. negrelle@ufpr.br
\end{abstract}

Recebido para publicação em 1/07/2011 e aceito em 13/08/2014 
were decíduos; $23 \%$ semideciduous and $26 \%$ evergreen. Attalea phalerata was the species with the highest structural value $(\mathrm{VI}=121.62)$, due to its high density ( $64 \%$ of the total sampled individuals), frequency $(100 \%)$ e and very high basal area $\left(74.10 \mathrm{~m}^{2} \mathrm{ha}^{-1}\right)$. On second level of importance were Anadenanthera colubrina, Triplaris americana and Phyllostylon rhamnoide (VI=35.41; 28.51; 18.5 respectively). The studied area was identified as very peculiar comparatively with other Seasonal Forest areas, especially because the Attalea phalerata massive density and absence of Myrtaceae representatives. The high representation of pioneer species in association with high density of individuals within this successional category reinforces that Mata com Acuri represents an early successional stage or a transitional stage. Based on the great percentage of deciduous and semi-deciduous species recorded for the area of study, it was classified as being representative of Deciduous Forest.

Keywords: Seasonal Deciduous Alluvial Forest; floristic; phytosociology.

\section{INTRODUÇÃO}

A paisagem do Pantanal é muito heterogênea, resultado de uma variedade de unidades geomorfológicas de pequena escala, em combinação com inundações anuais que criam uma grande diversidade de habitat (CUNHA; JUNK, 2004). Essa heterogeneidade também resulta do encontro de quatro províncias biogeográficas: Cerrado, Amazônica, Chaquenha e Atlântica (CABRERA; WILLINK, 1980; CORDEIRO, 2004; POTT et al., 2011).

Pelo menos quatro regiões fitoecológicas podem ser evidenciadas nesta região: a Savana (Cerrado), ocupando os relevos conservados e dissecados dos planaltos e as áreas de acumulação inundáveis; a Savana Estépica (vegetação chaquenha), ocorrendo em áreas de acumulação inundáveis entre o rio Paraguai e a linha de fronteira com a Bolívia em solos predominantemente salinos; a Floresta Estacional Semidecidual, ocupando as Planícies Aluviais e os terraços dos rios que compõem a rede hidrográfica da área; e a Floresta Estacional Decidual, observada em áreas de relevo dissecado (Paleozoico, Pré-Cambriano). Registramse adicionalmente Áreas de Tensão Ecológica e áreas submetidas à ação antrópica em diferentes posicionamentos geomorfológicos (BRASIL, 1982; POTT et al., 2011).

A Floresta Estacional Semidecidual (FESD) integra a vegetação típica do bioma da Mata Atlântica, interiorizando-se até a porção mais oriental do País, estando condicionada pela sazonalidade climática, perdendo parte das folhas (20 a 50\%) nos períodos secos. É constituída por fanerófitos com gemas foliares protegidas da seca por escamas (catáfilos ou pelos), tendo folhas adultas esclerófilas ou membranáceas deciduais. $\mathrm{O}$ grau de deciduidade, ou seja, a perda das folhas é dependente da intensidade e duração de basicamente duas razões: as temperaturas mínimas máximas e a deficiência do balanço hídrico (IBGE, 1992).

A Floresta Estacional Decidual (FED) é caracterizada também por duas estações climáticas bem marcadas, com longo período biologicamente seco. Ocorre na forma de disjunções florestais apresentando o estrato dominante macro ou mesofanerófito predominantemente caducifólio, com mais de $50 \%$ dos indivíduos despidos de folhagem no período desfavorável (IBGE, 1992).

No Pantanal, tanto na FESD quanto na FED, registra-se a ocorrência de Attalea phalerata Mart. ex Spreg., mesclada com espécies arbóreas em distintas densidades e podendo formar densas aglomerações monoespecíficas conhecidas como Acurizal (POTT et al., 2011). Em geral, a ocorrência densa de Attalea phalerata (acuri) no sub-bosque florestal pantaneiro caracteriza a chamada Mata com Acuri (HOFMANN et al., 2010).

Attalea phalerata é uma palmeira neotropical de distribuição restrita à porção oriental da América do Sul, sendo encontrada na Colômbia, Peru, Bolívia, Brasil e Paraguai (MISSOURI BOTANICAL GARDEN, 2011). Esta palmeira é considerada como indicativa de solos férteis (LORENZI, 1992). Cresce quase que exclusivamente em solos argilosos de ótima fertilidade e ricos em cálcio. Em algumas regiões, tem-se o hábito de deixálos nos pastos para valorizar as terras. Dificilmente ocorre em solos arenosos (POTT; POTT, 1994). Esta espécie é citada como um recurso-chave nas áreas onde ocorre, constituindo uma fonte de energia tanto para animais especialistas como generalistas (TERBORGH, 1986). É também considerada como espécie de elevado potencial econômico, dada a diversidade de usos populares a ela associados, 
incluindo emprego como fonte alimentar, recursos forrageiros, material para construções (GUARIM NETO, 1992; MIRANDA et al., 2001).

Apesar dos inúmeros estudos florísticos e estruturais realizados no Pantanal (e.g. POTT; POTT, 1994; NUNES DA CUNHA; UNK, 2001; DAMASCENO-JUNIOR et al., 2009; entre outros), especificamente no que se refere à Mata com Acuri as informações ainda são escassas. Dentre os poucos estudos aí realizados, incluem-se monitoramento da fenologia de acuri (SALIS et al., 1999); avaliação disponibilidade de frutos e dispersão de acuri (NASCIMENTO et al., 2004); distribuição espacial de acuri em relação aos tipos de solo (LIMA JUNIOR et al., 2007); presença de artrópodes em copas de acuri (SANTOS et al., 2003); biomassa de acuri (SALIS et al., 2007) e avaliação do microclima (HOFMANN et al., 2010).

Visando contribuir para o maior conhecimento deste tipo florestal pantaneiro, apresenta-se resultado de levantamento florísticoestrutural realizado em área de Mata com Acuri. Os dados obtidos foram contrastados com o reportado na literatura para FESD e FED de outras localidades, buscando evidenciar similitudes e diferenças.

\section{MATERIAL E MÉTODO}

\section{Local de estudo}

Otrabalho foirealizadona Reserva Particular do Patrimônio Natural (RPPN) SESC Pantanal, que abrange 1.076 quilômetros quadrados, localizada no município de Barão de Melgaço, no sul de Mato Grosso $\left(16^{\circ} 45^{\prime} \mathrm{S}\right.$ e $\left.56^{\circ} 15^{\prime} \mathrm{W}\right)$. A área encontra-se próxima ao centro geográfico da América do Sul, no noroeste do Pantanal, uma planície sedimentar que forma uma das maiores extensões úmidas e contínuas da Terra (HAMILTON et al., 1996). Apresenta um período de inundação entre os meses de dezembro a abril, devido ao acúmulo de chuvas na região e nas cabeceiras dos rios da Bacia do Alto Paraguai. O clima da região é típico de Savana, do tipo "Aw" segundo a classificação de Köppen, com a precipitação concentrando-se no verão, em um total anual que varia entre 1.000 e 1.600 milímetros.

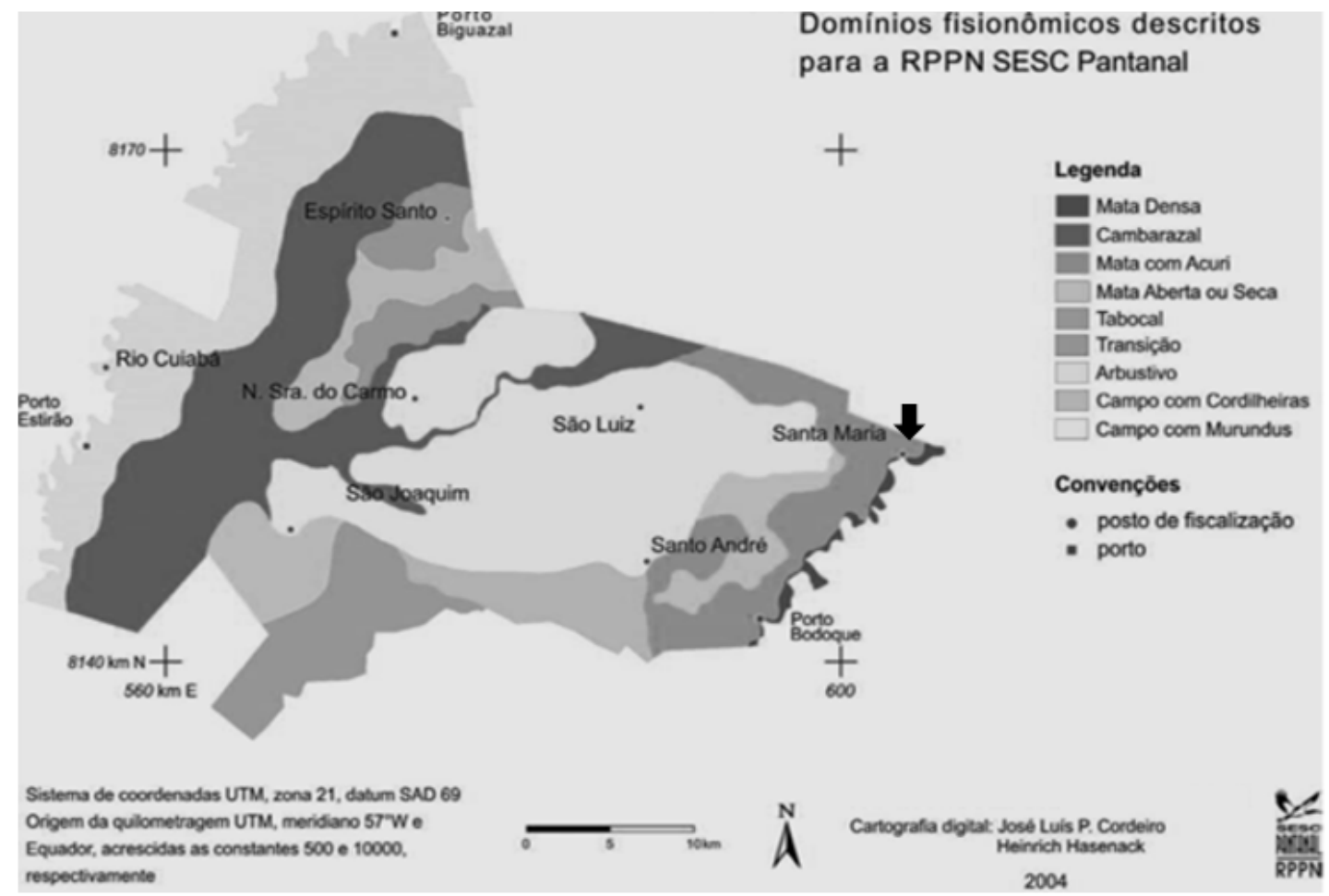

FIGURA 1: Cobertura do solo na RPPN SESC Pantanal. A flecha indica a área de Mata com Acuri onde o estudo florístico-estrutural foi realizado. (Fonte: SESC, 2010).

FIGURE 1: RPPN SESC Pantanal soil covertures, with an arrow indicating the Acuri Forest were the floristic-structural study was performed. (Source: SESC, 2010). 
No inverno predomina o clima seco, em decorrência da estabilidade gerada pela influência do anticiclone subtropical do Atlântico Sul e de pequenas dorsais que se formam sobre o continente (NIMER, 1989). A temperatura média anual é de $26,5^{\circ} \mathrm{C}$, com as médias mensais nunca inferiores a $22^{\circ} \mathrm{C}$ (HOFMANN et al., 2010).

A pesquisa foi desenvolvida em área de um ha de Mata com Acuri (Figura 1), com evidências visuais e registro histórico de ocorrência ocasional de fogo e corte raso há 25 anos atrás. O solo desta área foi caracterizado como Plintossolo Háplico Alítico solódico (BEIRIGO et al., 2011).

\section{Coleta de dados}

Os dados florísticos foram obtidos a partir de coletas sistemáticas de material botânico fértil e vegetativo efetuadas na área amostral e entorno desta. A identificação do material botânico coletado seguiu padrões de taxonomia clássica, feita com base em caracteres morfológicos florais ou vegetativos. As determinações foram efetuadas por meio de chaves analíticas e comparações com materiais depositados em diferentes herbários e quando possível enviado a especialistas para confirmação e/ou identificação. O material identificado foi formalmente depositado nos Herbários da Universidade Federal do Paraná UPCB (Curitiba) e da Universidade Estadual de Maringá NUPELIA (Maringá).

Para coleta de dados estruturais, a área foi subdividida em 100 parcelas contíguas de $10 \mathrm{~m} \mathrm{x}$ $10 \mathrm{~m}$. Dentro de cada uma destas parcelas, todos os indivíduos arbóreos com diâmetro a altura do peito igual ou maior que $5,0 \mathrm{~cm}$ e palmeiras foram identificados, mensurados e mapeados, utilizandose o sistema de coordenadas. Registrou-se a altura total (base do tronco ao final da copa) e o DAP de todos os indivíduos amostrados. Para as palmeiras, efetuou-se a medida da altura do estipe até o ponto de inserção das folhas e do diâmetro da base do estipe ( $\mathrm{DB} \geq 5 \mathrm{~cm}$ ). Estes registros foram utilizados para quantificar: Frequência, Densidade, Área Basal, Dominância e Valor de Importância (sensu MUELLER-DOMBOIS; ELLENBERG, 1974).

Para avaliação da estratificação vegetal, utilizou-se a classificação de alturas: macrofanerófitos $(30 \mathrm{~m})$, mesofanerófitos $(20<30$ $\mathrm{m})$, microfanerófitos $(5<20 \mathrm{~m})$ e nanofanerófitos $(>0,25<5 \mathrm{~m})($ IBGE, 1992).

Para o cálculo da diversidade foram considerados dois índices de diversidade alfa: $\mathrm{S}$ (riqueza florística) e índice de Shannon, conforme Magurran (1987).

A confirmação e atualização da nomenclatura botânica foram realizadas em Missouri Botanical Garden (2011). Os dados relativos aos domínios fitogeográficos das espécies foram obtidos em Forza et al. (2010). As informações relativas à deciduidade e status sucessional destas espécies foram obtidas a partir de revisão de literatura.

A comparação fisionômica e florística com outras florestas similares foi efetuada com base em dados bibliográficos de trabalhos que utilizaram critérios semelhantes aos empregados neste estudo. Utilizou-se o Índice de Sørensen binário para calcular a similaridade entre distintos sítios, conforme Mueller-Dombois e Ellenberg (1974).

\section{RESULTADOS}

Foram amostrados 1224 indivíduos. ha $^{-1}$, representativos de 44 espécies, 43 gêneros e 27 famílias (Tabela 1), índice de diversidade H' $=1,613$ (var. 0,003514). As famílias identificadas com maior diversidade na área foram Fabaceae ( 9 spp.), Sapindaceae e Anacardiaceae (3 spp. cada), Bignoniaceae, Malvaceae, Moraceae e Rubiaceae (2 spp. cada). As demais famílias estavam representadas por apenas uma espécie.

Dentre as espécies amostradas, 39\% eram perenifólias; $32 \%$ semidecíduas e $29 \%$ decíduas. Do total de indivíduos arbóreos $(\mathrm{n}=440), 51 \%$ foram identificados como decíduos; $23 \%$ semidecíduos e $26 \%$ perenifólios.

Registrou-se $\quad 39 \% \quad$ de espécies categorizadas como pioneiras, sendo as demais identificadas como não pioneiras. Do total de indivíduos amostrados $(\mathrm{n}=1224), \quad 96 \%$ eram representantes de espécies categorizadas como pioneiras.

A grande maioria das espécies amostradas apresenta padrão amplo de distribuição, ocorrendo em vários biomas brasileiros, especialmente Amazônia, Cerrado, Pantanal e Mata Atlântica (Figura 2).

No geral, os indivíduos amostrados apresentavam porte relativamente pouco desenvolvido, tanto em altura como diâmetro, porém, não se detectou correlação significativa entre estas duas variáveis $(\mathrm{r}=0,2676 ; \mathrm{p}=0,0790)$. $\mathrm{O}$ diâmetro médio registrado foi $14,45 \pm 10,07 \mathrm{~cm}$ $($ máximo $=48,8 \mathrm{~cm}$; mínimo $=5,35 \mathrm{~cm}) . A$ área basal total amostrada foi $88,43 \mathrm{~m}^{2} \mathrm{ha}^{-1}$, sendo $84 \%$ 


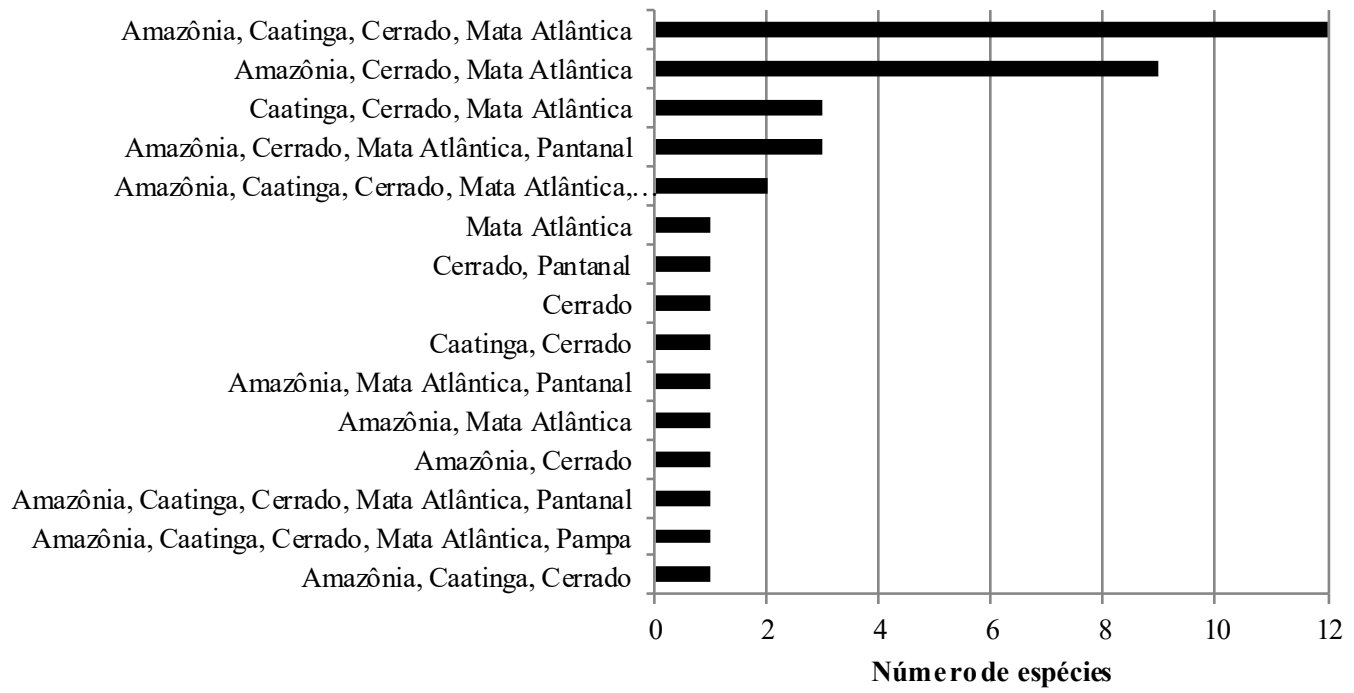

FIGURA 2: Domínios fitogeográficos associados à distribuição das espécies registradas em área (1 ha) classificada como "Mata com Acuri” (RPPN SESC Pantanal, Município de Barão de Melgaço - MT).

FIGURE 2: Phytogeographic domains associated with the distribution of the species registered in 1 ha plot classified as “Acuri Forest” (RPPN SESC Pantanal, Municipality of Barão de Melgaço - MT).

deste valor correspondente à área basal de apenas uma espécie: Attalea phalerata Mart. ex Spreg.

A altura média correspondeu a $8,62 \pm$ 3,26 m (máx. =20,5 m; mín. =2,5 m). A maioria das espécies amostradas (80\%) enquadrava-se como mesofanerófita, havendo reduzida participação de espécies nanofanerófitas $(2 \%)$ e macrofanerófitas (18\%). Em função do nível de ocupação do eixo vertical, detectaram-se três categorias de espécies: emergentes $(\geq 17 \mathrm{~m})$, dossel $(\geq 10<17 \mathrm{~m})$ e subdossel $(<10 \mathrm{~m})$. Na condição de emergentes, identificaram-se Hymenaea courbaril; Spondias mombin; Astronium fraxinifolium; Anadenanthera colubrina; Cassia grandis. Comparativamente, o dossel e subdossel apresentavam riqueza bem mais elevada, 21 e 18 espécies respectivamente (Figura 3). No subdossel, registrou-se predominância massiva de representantes de Attalea phalerata.

A grande maioria das espécies amostradas (77,3\%) estava representada por menos de 10 indivíduos e apenas quatro espécies apresentaram densidade superior a 50 indivíduos. Em geral, dada sua baixa densidade, as espécies estavam distribuídas de maneira bastante setorizada na área avaliada, sendo que $79 \%$ apresentavam-se restritas a menos de $10 \%$ da área e apenas $4 \%$ ocorriam dispersas em mais de $50 \%$ desta.
Attalea phalerata foi a espécie com maior valor de importância estrutural $(\mathrm{VI}=121,62)$, devido à sua alta densidade $(64 \%$ do total de indivíduos amostrados), frequência (100\%) e elevadíssima área basal. Em segundo nível de importância registraram-se Anadenanthera colubrina, Triplaris americana e Phyllostylon rhamnoide (VI $=35,41$; 28,$51 ; 18,51$ respectivamente). O VI obtido para Anadenanthera colubrina foi especialmente devido à sua elevada área basal. Os VI de Triplaris americana e Phyllostylon rhamnoide foram devidos às suas respectivas altas densidades. Em terceiro nível, englobaram-se as demais espécies cujo valor de importância conjunto correspondeu a $27 \%$ do valor de importância total (300) (Tabela 1).

A comparação florístico-estrutural entre remanescentes de FESD revelou grande heterogeneidade entre estes e reforçou a particularidade da Mata com Acuri como formação pioneira, com relativa baixa diversidade acompanhada de elevada área basal. Detectouse reduzida similaridade florística entre a área estudada e outros sítios representativos de FESD (Tabela 3). Em termos florísticos, detectou-se nível mais elevado de similaridade apenas com área reportada em Romagnolo e Souza (2000). No entanto, neste não há registro de ocorrência 
de Attalea phalerata. A presença desta espécie só é reportada em Salis et al. (2004), porém, com densidade bem inferior.

\section{DISCUSSÃO}

A heterogeniedade de critérios de inclusão e área amostral aplicados nos distintos trabalhos já realizados em FESD e FED dificulta a completa comparação entre sítios, especialmente no que concerne a dados estruturais. No entanto, evidenciou-se grande peculiaridade para a área estudada, especialmente pela expressiva densidade de Attalea phalerata, que acabou por influenciar vários dos parâmetros analisados, incluindo redução do índice de diversidade $\left(\mathrm{H}^{\prime}\right)$, elevação da porcentagem de indivíduos perenifólios e elevação da área basal total. Ou seja, ao se considerar a área analisada excluindo-se esta palmeira, obtêm-se valores destes parâmetros similares aos reportados para outros sítios de FESD e FED, como também reportado em Duarte (2007).

Outro aspecto diferenciado da área estudada foi a ausência de representantes de Myrtaceae, reportada entre as famílias de maior diversidade em vários sítios representativos de FESD, especialmente aquelas mais próximas da costa atlântica (ver p.ex. IVANAUSKAS et al., 1999; MEIRA-NETO; MARTINS, 2002; LOPES et al., 2011, entre outros). Apesar da grande maioria das espécies amostradas apresentarem padrão amplo de distribuição, aparentemente a área estudada sofre maior influência das províncias biogeográficas Cerrado, Amazônica e Chaquenha, em detrimento da Província Atlântica na qual a Myrtaceae destacase como elemento de caracterização. A análise comparativa com os resultados apresentados em Dislich et al. (2001) reforça esta característica diferenciada da área analisada e a pouca semelhança com outros sítios de menor continentalidade.

A elevada representatividade de espécies pioneiras (39\%) em associação à elevada densidade de indivíduos dentro desta categoria sucessional reforça o caráter de formação transicional da Mata com Acuri. Esta formação vegetal é tida como fase intermediária entre as formações mais abertas ou semiabertas para as de dossel mais fechado da FESD pantaneira (Mata Densa) (HOFMANN et al., 2010; NEGRELLE, 2013). Attalea phalerata, espécie característica desteambiente, prefere asáreas abertas, sendo sua presença considerada como indicação de solos férteis. Cresce quase que exclusivamente em solos argilosos de ótima fertilidade e ricos em cálcio. Também Anadenanthera colubrina e Triplaris americana são espécies decíduas, heliófitas, pioneiras de crescimento rápido que caracterizam áreas secundárias de solos argilosos (LORENZI, 1992). Entretanto, é questionável a classificação de Hofmann et al. (2010) para a Mata com Acuri considerando-a como representativa de FESD.

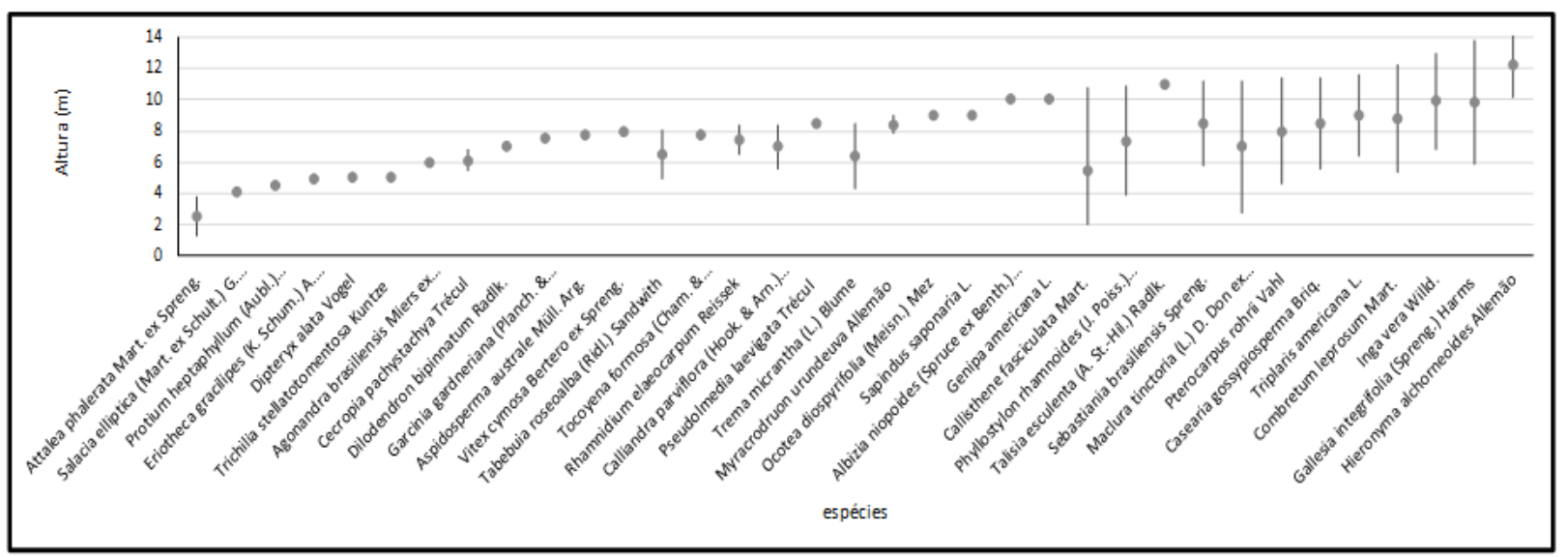

FIGURA 3: Diagrama da estratificação vertical (alturas mínima, média e máxima) das espécies amostradas em área (1 ha) classificada como "Mata com Acuri" (RPPN SESC Pantanal, Município de Barão de Melgaço - MT).

FIGURE 3: Vertical stratification diagram (minimum, average and maximum height) of the species registered in 1 ha plot classified as "Acuri Forest" (RPPN SESC Pantanal, Municipality of Barão de Melgaço - MT). 
TABELA 1: Espécies amostradas em área (1 ha) classificada como "Mata com Acuri" (RPPN SESC Pantanal, Município de Barão de Melgaço - MT) e respectivos parâmetros fitossociológicos, em ordem decrescente de valor da importância (VI).

TABLE 1: Species registered in 1 ha plot classified as "Acuri Forest" (RPPN SESC Pantanal, Municipality of Barão de Melgaço - MT) with respective phytosociological parameters, in decreasing order of importance value (VI).

\begin{tabular}{|c|c|c|c|c|c|c|c|}
\hline & DA & $\mathrm{DR}$ & FA & FR & DoA & DoR & VI \\
\hline Attalea phalerata Mart. ex Spreng. & 784 & 64,05 & 100 & 23,53 & 19963,01 & 83,92 & 171,50 \\
\hline Triplaris americana $\mathrm{L}$. & 106 & 8,66 & 66 & 15,53 & 9,82 & 0,04 & 24,23 \\
\hline Anadenanthera colubrina (Vell.) Brenan & 61 & 4,98 & 35 & 8,24 & 1363,7 & 5,73 & 18,95 \\
\hline Phyllostylon rhamnoides (J. Poiss.) Taub. & 54 & 4,41 & 35 & 8,24 & 552,96 & 2,32 & 14,97 \\
\hline Combretum leprosum Mart. & 42 & 3,43 & 29 & 6,82 & 544,08 & 2,29 & 12,54 \\
\hline Sebastiania brasiliensis Spreng. & 34 & 2,78 & 31 & 7,29 & 13,09 & 0,06 & 10,13 \\
\hline Gallesia integrifolia (Spreng.) Harms & 20 & 1,63 & 17 & 4,00 & 351,99 & 1,48 & 7,11 \\
\hline Tabebuia roseoalba (Ridl.) Sandwith & 20 & 1,63 & 19 & 4,47 & 9,68 & 0,04 & 6,15 \\
\hline Spondias mombin L. & 13 & 1,06 & 10 & 2,35 & 38,85 & 0,16 & 3,58 \\
\hline Callisthene fasciculata Mart. & 8 & 0,65 & 8 & 1,88 & 118,22 & 0,50 & 3,03 \\
\hline Inga vera Willd. & 7 & 0,57 & 7 & 1,65 & 118,66 & 0,50 & 2,72 \\
\hline Casearia gossypiosperma Briq. & 10 & 0,82 & 6 & 1,41 & 67,98 & 0,29 & 2,51 \\
\hline Trema micrantha (L.) Blume & 7 & 0,57 & 5 & 1,18 & 8,17 & 0,03 & 1,78 \\
\hline Hieronyma alchorneoides Allemão & 4 & 0,33 & 4 & 0,94 & 62,53 & 0,26 & 1,53 \\
\hline Astronium fraxinifolium Schott ex Spreng. & 4 & 0,33 & 4 & 0,94 & 55,3 & 0,23 & 1,50 \\
\hline Pterocarpus rohrii Vahl & 5 & 0,41 & 4 & 0,94 & 33,84 & 0,14 & 1,49 \\
\hline Tabebuia serratifolia (Vahl) G. Nicholson & 4 & 0,33 & 4 & 0,94 & 21,55 & 0,09 & 1,36 \\
\hline Guazuma ulmifolia Lam. & 3 & 0,25 & 3 & 0,71 & 40,9 & 0,17 & 1,12 \\
\hline Cecropia pachystachya Trécul & 3 & 0,25 & 3 & 0,71 & 13,66 & 0,06 & 1,01 \\
\hline Mouriri guianensis Aubl. & 2 & 0,16 & 2 & 0,47 & 87,14 & 0,37 & 1,00 \\
\hline Rhamnidium elaeocarpum Reissek & 3 & 0,25 & 3 & 0,71 & 8,53 & 0,04 & 0,99 \\
\hline Cassia grandis L. f. & 2 & 0,16 & 2 & 0,47 & 76,62 & 0,32 & 0,96 \\
\hline Hymenaea courbaril L. & 2 & 0,16 & 2 & 0,47 & 28,82 & 0,12 & 0,76 \\
\hline Maclura tinctoria (L.) D. Don ex Steud. & 2 & 0,16 & 2 & 0,47 & 16,41 & 0,07 & 0,70 \\
\hline Talisia esculenta (A. St.-Hil.) Radlk. & 2 & 0,16 & 2 & 0,47 & 15,6 & 0,07 & 0,70 \\
\hline Tocoyena formosa (Cham. \& Schltdl.) K. Schum. & 2 & 0,16 & 2 & 0,47 & 11 & 0,05 & 0,68 \\
\hline Salacia elliptica (Mart. ex Schult.) G. Don & 2 & 0,16 & 2 & 0,47 & 5,35 & 0,02 & 0,66 \\
\hline Myracrodruon urundeuva Allemão & 1 & 0,08 & 2 & 0,47 & 15,7 & 0,07 & 0,62 \\
\hline Acacia paniculata Willd. & 2 & 0,16 & 1 & 0,24 & 16,88 & 0,07 & 0,47 \\
\hline Machaerium acutifolium Vogel & 1 & 0,08 & 1 & 0,24 & 14,92 & 0,06 & 0,38 \\
\hline Rheedia brasiliensis (Mart.) Planch. \& Triana & 1 & 0,08 & 1 & 0,24 & 11,5 & 0,05 & 0,37 \\
\hline Pseudolmedia laevigata Trécul & 1 & 0,08 & 1 & 0,24 & 10,99 & 0,05 & 0,36 \\
\hline Genipa americana $\mathrm{L}$. & 1 & 0,08 & 1 & 0,24 & 10,6 & 0,04 & 0,36 \\
\hline Aspidosperma australe Müll. Arg. & 1 & 0,08 & 1 & 0,24 & 9,42 & 0,04 & 0,36 \\
\hline Sapindus saponaria $\mathrm{L}$. & 1 & 0,08 & 1 & 0,24 & 9,4 & 0,04 & 0,36 \\
\hline Protium heptaphyllum (Aubl.) Marchand & 1 & 0,08 & 1 & 0,24 & 8,87 & 0,04 & 0,35 \\
\hline
\end{tabular}


TABELA1: Continuação...

TABLE 1: Continued...

\begin{tabular}{lccccccc}
\hline & DA & DR & FA & FR & DoA & DoR & VI \\
\hline Agonandra brasiliensis Miers ex Benth. \& Hook. f. & 1 & 0,08 & 1 & 0,24 & 7,77 & 0,03 & 0,35 \\
Trichilia stellatotomentosa Kuntze & 1 & 0,08 & 1 & 0,24 & 6,5 & 0,03 & 0,34 \\
Ocotea diospyrifolia (Meisn.) Mez & 1 & 0,08 & 1 & 0,24 & 6,44 & 0,03 & 0,34 \\
Albizia niopoides (Spruce ex Benth.) Burkart & 1 & 0,08 & 1 & 0,24 & 6,28 & 0,03 & 0,34 \\
Dilodendron bipinnatum Radlk. & 1 & 0,08 & 1 & 0,24 & 5,42 & 0,02 & 0,34 \\
Dipteryx alata Vogel & 1 & 0,08 & 1 & 0,24 & 5,22 & 0,02 & 0,34 \\
Eriotheca gracilipes (K. Schum.) A. Robyns & 1 & 0,08 & 1 & 0,24 & 4,4 & 0,02 & 0,34 \\
Vitex cymosa Bertero ex Spreng. & 1 & 0,08 & 1 & 0,24 & 10,5, & 0,00 & 0,32 \\
\hline
\end{tabular}

Em que: $\mathrm{DA}=$ densidade absoluta (n. indivíduos.ha $\left.{ }^{-1}\right) ; \mathrm{DR}=$ densidade relativa (\%); $\mathrm{FA}=$ frequência absoluta; $\mathrm{FR}=$ frequência relativa (\%); DoA= dominância absoluta $\left(\mathrm{m}^{2}\right)$; DoR= Dominância relativa $(\%)$.

TABELA 2: Espécies amostradas em área (1 ha) classificada como "Mata com Acuri" (RPPN SESC Pantanal, Município de Barão de Melgaço $-\mathrm{MT}$ ) e respectivas famílias, DEC = deciduidade $(\mathrm{P}=$ perenifólia; $\mathrm{SD}=$ semidecídua; $\mathrm{D}=$ decídua $) ; \mathrm{S} . \mathrm{S}=$ status sucessional $(\mathrm{P}=$ pioneira; $\mathrm{NP}=$ não pioneira) e distribuição geográfica.

TABLE 2: Species registered in 1 ha plot classified as "Acuri Forest" (RPPN SESC Pantanal, Municipality of Barão de Melgaço - MT) with respective families; DEC = Deciduousness $(\mathrm{P}=$ evergreen; $\mathrm{SD}=$ semideciduous; $\mathrm{D}=$ deciduous $) . \mathrm{S} . \mathrm{S}=$ sucessional status $(\mathrm{P}=$ pioneer; $\mathrm{NP}=$ not pioneer) and, geographic distribution .

\begin{tabular}{|c|c|c|c|c|c|}
\hline Família & Espécie & $\mathrm{DEC}$ & S.S. & Distribuição & \\
\hline Anacardiaceae & Astronium fraxinifolium Schott ex Spreng. & $\mathrm{D}$ & $\mathrm{P}$ & Amazônia, Cerrado, Mata Atlântica & \\
\hline Anacardiaceae & Myracrodruon urundeuva Allemão & $\mathrm{D}$ & NP & $\begin{array}{l}\text { Amazônia, Caatinga, Cerrado, } \\
\text { Atlântica, Pantanal }\end{array}$ & Mata \\
\hline Anacardiaceae & Spondias mombin $\mathrm{L}$. & $\mathrm{P}$ & $\mathrm{P}$ & Cerrado & \\
\hline Apocynaceae & Aspidosperma australe Müll. Arg. & $\mathrm{S}$ & NP & Amazônia, Cerrado, Mata Atlântica & \\
\hline Arecaceae & Attalea phalerata Mart. ex Spreng. & $\mathrm{P}$ & $\mathrm{P}$ & Caatinga, Cerrado, Mata Atlântica & \\
\hline Bignoniaceae & Handroanthus serratifolius (Vahl) S. O. Grose & $\mathrm{D}$ & NP & Caatinga, Cerrado, Mata Atlântica & \\
\hline Bignoniaceae & Tabebuia roseoalba (Ridl.) Sandwith & $\mathrm{D}$ & NP & $\begin{array}{l}\text { Amazônia, Caatinga, Cerrado, } \\
\text { Atlântica, Pampa, Pantanal }\end{array}$ & Mata \\
\hline Burseraceae & Protium heptaphyllum (Aubl.) Marchand & $\mathrm{P}$ & NP & $\begin{array}{l}\text { Amazônia, Caatinga, Cerrado, } \\
\text { Atlântica, Pampa }\end{array}$ & Mata \\
\hline Cannabaceae & Trema micrantha (L.) Blume & $\mathrm{P}$ & $\mathrm{P}$ & $\begin{array}{l}\text { Amazônia, Caatinga, Cerrado, } \\
\text { Atlântica }\end{array}$ & Mata \\
\hline Celastraceae & Salacia elliptica (Mart. ex Schult.) G. Don & $P$ & NP & Amazônia, Caatinga, Cerrado & \\
\hline Clusiaceae & Garcinia gardneriana (Planch. \& Triana) Zappi & $\mathrm{P}$ & NP & Amazônia, Cerrado, Mata Atlântica & \\
\hline Combretaceae & Combretum leprosum Mart. & S & NP & Amazônia, Cerrado, Mata Atlântica & \\
\hline Euphorbiaceae & Sebastiania brasiliensis Spreng. & $\mathrm{S}$ & NP & Amazônia, Cerrado, Mata Atlântica & \\
\hline Fabaceae & Albizia niopoides (Spruce ex Benth.) Burkart & $\mathrm{S}$ & $\mathrm{P}$ & $\begin{array}{l}\text { Amazônia, Caatinga, Cerrado, } \\
\text { Atlântica }\end{array}$ & Mata \\
\hline Fabaceae & Anadenanthera colubrina (Vell.) Brenan & $\mathrm{D}$ & $\mathrm{P}$ & Caatinga, Cerrado, Mata Atlântica & \\
\hline Fabaceae & Calliandra parviflora (Hook. \& Arn.) Speg. & $\mathrm{P}$ & $\mathrm{P}$ & $\begin{array}{l}\text { Amazônia, Caatinga, Cerrado, } \\
\text { Atlântica, Pantanal }\end{array}$ & Mata \\
\hline
\end{tabular}


TABELA 2: Continuação...

TABLE 2: Continued...

\begin{tabular}{|c|c|c|c|c|c|c|}
\hline Família & Espécie & DEC & S.S. & \multicolumn{3}{|l|}{ Distribuição } \\
\hline Fabaceae & Cassia grandis L. f. & $\mathrm{D}$ & $\mathrm{P}$ & \multicolumn{3}{|l|}{ Mata Atlântica } \\
\hline Fabaceae & Dipteryx alata Vogel & $P$ & NP & $\begin{array}{l}\text { Amazônia, Caatinga, } \\
\text { Atlântica }\end{array}$ & Cerrado, & Mata \\
\hline Fabaceae & Hymenaea courbaril L. & $\mathrm{S}$ & NP & $\begin{array}{l}\text { Amazônia, Caatinga, } \\
\text { Atlântica }\end{array}$ & Cerrado, & Mata \\
\hline Fabaceae & Inga vera Willd. & $\mathrm{S}$ & $\mathrm{P}$ & \multicolumn{3}{|l|}{ Caatinga, Cerrado } \\
\hline Fabaceae & Machaerium acutifolium Vogel & $\mathrm{S}$ & NP & \multicolumn{3}{|c|}{ Amazônia, Mata Atlântica, Pantanal } \\
\hline Fabaceae & Pterocarpus rohrii Vahl & $\mathrm{P}$ & $\mathrm{P}$ & $\begin{array}{l}\text { Amazônia, Caatinga, } \\
\text { Atlântica }\end{array}$ & Cerrado, & Mata \\
\hline Lamiaceae & Vitex cymosa Bertero ex Spreng. & $\mathrm{D}$ & NP & $\begin{array}{l}\text { Amazônia, Caatinga, } \\
\text { Atlântica, Pantanal }\end{array}$ & Cerrado, & Mata \\
\hline Lauraceae & Ocotea diospyrifolia (Meisn.) Mez & $P$ & NP & $\begin{array}{l}\text { Amazônia, Caatinga, } \\
\text { Atlântica }\end{array}$ & Cerrado, & Mata \\
\hline Malvaceae & Eriotheca gracilipes (K. Schum.) A. Robyns & $\mathrm{S}$ & NP & $\begin{array}{l}\text { Amazônia, Caatinga, } \\
\text { Atlântica }\end{array}$ & Cerrado, & Mata \\
\hline Malvaceae & Guazuma ulmifolia Lam. & $\mathrm{S}$ & $P$ & $\begin{array}{l}\text { Amazônia, Cerrado, } \\
\text { Pantanal }\end{array}$ & Mata Atlâ & întica, \\
\hline Melastomataceae & Mouriri guianensis Aubl. & $\mathrm{P}$ & NP & Cerrado, Pantanal & & \\
\hline Meliaceae & Trichilia stellatotomentosa Kuntze & $\mathrm{P}$ & NP & $\begin{array}{l}\text { Amazônia, Caatinga, } \\
\text { Atlântica }\end{array}$ & Cerrado, & Mata \\
\hline Moraceae & Maclura tinctoria (L.) D. Don ex Steud. & $\mathrm{D}$ & $\mathrm{P}$ & $\begin{array}{l}\text { Amazônia, Caatinga, } \\
\text { Atlântica, Pantanal }\end{array}$ & Cerrado, & Mata \\
\hline Moraceae & Pseudolmedia laevigata Trécul & $\mathrm{P}$ & NP & Amazônia, Cerrado, Mat & ta Atlântica & \\
\hline Opiliaceae & $\begin{array}{l}\text { Agonandra brasiliensis Miers ex Benth. \& } \\
\text { Hook. f. }\end{array}$ & $\mathrm{D}$ & NP & $\begin{array}{l}\text { Amazônia, Caatinga, } \\
\text { Atlântica, Pantanal }\end{array}$ & Cerrado, & Mata \\
\hline Phyllanthaceae & Hieronyma alchorneoides Allemão & $\mathrm{S}$ & NP & Amazônia, Cerrado, Ma & ta Atlântica & \\
\hline Phytolaccaceae & Gallesia integrifolia (Spreng.) Harms & $\mathrm{P}$ & NP & $\begin{array}{l}\text { Amazônia, Cerrado, } \\
\text { Pantanal }\end{array}$ & Mata Atlâ & ântica, \\
\hline Polygonaceae & Triplaris americana $\mathrm{L}$. & $\mathrm{D}$ & $\mathrm{P}$ & $\begin{array}{l}\text { Amazônia, Caatinga, } \\
\text { Atlântica, Pantanal }\end{array}$ & Cerrado, & Mata \\
\hline Rhamnaceae & Rhamnidium elaeocarpum Reissek & $\mathrm{D}$ & $\mathrm{P}$ & $\begin{array}{l}\text { Amazônia, Caatinga, } \\
\text { Atlântica }\end{array}$ & Cerrado, & Mata \\
\hline Rubiaceae & Genipa americana $\mathrm{L}$. & $\mathrm{S}$ & NP & Amazônia, Cerrado, Mat & ta Atlântica & \\
\hline Rubiaceae & Tocoyena formosa (Cham. \& Schltdl.) K. Schum. & $\mathrm{P}$ & $\mathrm{P}$ & Amazônia, Cerrado & & \\
\hline Salicaceae & Casearia gossypiosperma Briq. & $\mathrm{D}$ & NP & $\begin{array}{l}\text { Amazônia, Caatinga, } \\
\text { Atlântica }\end{array}$ & Cerrado, & Mata \\
\hline Sapindaceae & Dilodendron bipinnatum Radlk. & $\mathrm{S}$ & $\mathrm{P}$ & $\begin{array}{l}\text { Amazônia, Caatinga, } \\
\text { Atlântica }\end{array}$ & Cerrado, & Mata \\
\hline Sapindaceae & Sapindus saponaria L. & $\mathrm{S}$ & NP & $\begin{array}{l}\text { Amazônia, Caatinga, } \\
\text { Atlântica, Pampa, Pantar }\end{array}$ & $\begin{array}{l}\text { Cerrado, } \\
\text { hal }\end{array}$ & Mata \\
\hline Sapindaceae & Talisia esculenta (A. St.-Hil.) Radlk. & $\mathrm{S}$ & NP & $\begin{array}{l}\text { Amazônia, Cerrado, } \\
\text { Pantanal }\end{array}$ & Mata Atlâ & ântica, \\
\hline Ulmaceae & Phyllostylon rhamnoides (J. Poiss.) Taub. & $\mathrm{P}$ & NP & Amazônia, Cerrado, Mat & ta Atlântica & \\
\hline Urticaceae & Cecropia pachystachya Trécul & $\mathrm{P}$ & $\mathrm{P}$ & $\begin{array}{l}\text { Amazônia, Caatinga, } \\
\text { Atlântica }\end{array}$ & Cerrado, & Mata \\
\hline Vochysiaceae & Callisthene fasciculata Mart. & $\mathrm{D}$ & NP & Amazônia, Mata Atlântic & & \\
\hline
\end{tabular}


TABELA 3: Dados florístico-estruturais de distintos sítios de Floresta Estacional Semidecidual, sendo: $\mathrm{S}=$ riqueza (número total de espécies); $\mathrm{N}$ = número total de indivíduos; $\mathrm{H}^{\prime}=$ índice de Shannon; $\mathrm{AB}=$ área basal; $\mathrm{VI}=$ valor de importância; $\mathrm{S}_{\text {sor }}=$ indíce de similaridade de $\mathrm{S} \varnothing$ rensen.

TABLE 3: Floristic-structural data from different sites representing Semideciduous Seasonal Forest, being: $\mathrm{S}=$ richness (total of species); $\mathrm{N}=$ total number of individuals; $\mathrm{H}^{\prime}=$ Shannon Index; $\mathrm{AB}=$ basal area; $\mathrm{VI}=$ importance value; $\mathrm{S}_{\text {sor }}=\mathrm{S} \varnothing$ rensen similarity index.

\begin{tabular}{|c|c|c|c|c|c|c|}
\hline & \multicolumn{6}{|c|}{ AUTOR } \\
\hline & Este trabalho & Salis et al., 2004 & $\begin{array}{l}\text { Arruda; } \\
\text { Daniel, } 2007\end{array}$ & $\begin{array}{l}\text { Romagnolo; } \\
\text { Souza, } 2000\end{array}$ & $\begin{array}{l}\text { Campos et al., } \\
2000\end{array}$ & $\begin{array}{l}\text { Cavassani, } \\
2007\end{array}$ \\
\hline Local & MT & MS & MS & MS & MS & PR \\
\hline Lat S/ & $16^{\circ} 45$ & $19^{\circ} 05^{\prime}$ & $22^{\circ} 13^{\prime}$ & $22^{\circ} 43^{\prime}$ & $22^{\circ} 43^{\prime}$ & $23^{\circ} 55^{\prime}$ \\
\hline Long W & $56^{\circ} 15^{\prime}$ & $57^{\circ} 40^{\prime}$ & $54^{\circ} 48^{\prime}$ & $53^{\circ} 15^{\prime}$ & $53^{\circ} 18^{\prime}$ & $51^{\circ} 56^{\prime}$ \\
\hline $\begin{array}{l}\text { Critério } \\
\text { inclusão }\end{array}$ & $\mathrm{DAP}>5 \mathrm{~cm}$ & $\begin{array}{l}\mathrm{CAP}>9 \mathrm{~cm} \\
(D A P>2,86 \mathrm{~cm})\end{array}$ & $\begin{array}{l}\mathrm{CAP}>15 \mathrm{~cm} \\
(D A P> \\
4,77 \mathrm{~cm})\end{array}$ & $\begin{array}{l}\mathrm{CAP}>15 \mathrm{~cm} \\
(D A P>4,77 \mathrm{~cm})\end{array}$ & $\begin{array}{l}\mathrm{CAP}>15 \mathrm{~cm} \\
(D A P>4,77 \mathrm{~cm})\end{array}$ & $\begin{array}{l}\mathrm{CAP}>10 \mathrm{~cm} \\
(D A P> \\
3,18 \mathrm{~cm})\end{array}$ \\
\hline Área & 1,0 ha & 20 ptos. & ni & 0,94 ha & 0,60 ha & 0,18 ha \\
\hline Riqueza & $\begin{array}{l}44 \mathrm{sp} \\
27 \mathrm{fam} \\
\end{array}$ & $32 \mathrm{sp}$ & $\begin{array}{l}76 \mathrm{sp} \\
29 \mathrm{fam} \\
\end{array}$ & $\begin{array}{l}56 \mathrm{sp} \\
30 \mathrm{fam} \\
\end{array}$ & $\begin{array}{l}47 \mathrm{sp} \\
28 \mathrm{fam}\end{array}$ & $50 \mathrm{sp}$ \\
\hline $\mathrm{N} / \mathrm{ha}$ & 1224 & 1020 & 1024 & 1877 & 1472 & 2266 \\
\hline $\mathrm{H}$ & 1,16 & $\mathrm{Ni}$ & 3,48 & 3,20 & 3,20 & $\mathrm{Ni}$ \\
\hline $\begin{array}{l}\mathrm{AB} \\
\mathrm{m}^{2} \mathrm{ha}^{-1}\end{array}$ & 88,47 & ni & 19,87 & 33,51 & 26,88 & $\mathrm{Ni}$ \\
\hline $\begin{array}{l}\text { Famílias } \\
\text { (maior } \\
\text { riqueza) }\end{array}$ & $\begin{array}{l}\text { Fabaceae (9) } \\
\text { Sapindaceae (3) } \\
\text { Anacardiaceae (3) }\end{array}$ & $\begin{array}{l}\text { Fabaceae (7) } \\
\text { Areceae (3) } \\
\text { Sapindaceae (3 }\end{array}$ & $\begin{array}{l}\text { Myrtaceae (8) } \\
\text { Lauraceae (8) } \\
\text { Mimosaceae } \\
(5)\end{array}$ & $\begin{array}{l}\text { Myrtaceae (5) } \\
\text { Lauracea (5) } \\
\text { Mimosaceae (4) }\end{array}$ & $\begin{array}{l}\text { Mimosaceae (4) } \\
\text { Lauraceae (4) } \\
\text { Caesalpinaceae (3) }\end{array}$ & $\begin{array}{l}\text { Fabaceae (7) } \\
\text { Myrtaceae (5) } \\
\text { Lauraceae (5) }\end{array}$ \\
\hline $\begin{array}{l}\text { Espécies } \\
\text { (maior VI) }\end{array}$ & $\begin{array}{l}\text { Attalea phalerata } \\
\text { Triplaris americana } \\
\text { Anadenanthera } \\
\text { colubrina } \\
\text { Phyllostylon } \\
\text { rhamnoides } \\
\text { Combretum } \\
\text { leprosum }\end{array}$ & $\begin{array}{l}\text { Attalea phalerata } \\
\text { Aspidosperma } \\
\text { australe } \\
\text { Copernicia alba } \\
\text { Pterogyne nitens } \\
\text { Chomelia obtusa }\end{array}$ & $n i$. & $\begin{array}{l}\text { Cecropia } \\
\text { pachystachia } \\
\text { Sloanea } \\
\text { guianensis } \\
\text { Guazuma } \\
\text { ulmifolia } \\
\text { Inga uruguensis } \\
\text { Peltoforum } \\
\text { dubium }\end{array}$ & $\begin{array}{l}\text { Cecropia } \\
\text { pachystachia } \\
\text { Inga uruguensis } \\
\text { Guazuma ulmifolia } \\
\text { Peltoforum dubium } \\
\text { Albizia hassleri }\end{array}$ & $N i$ \\
\hline S sor & ---- & 0,32 & 0,27 & 0,59 & 0,36 & 0,18 \\
\hline
\end{tabular}

Dada a elevada representatividade de indivíduos de espécies decíduas $(51 \%)$ e semidecíduas (23\%), conforme IBGE (1992), estes valores são mais indicativos de Floresta Decidual. Estudos específicos sobre este tema deverão esclarecer melhor esta questão.

Dada a peculiaridade da Mata com Acuri, no contexto da FESD e FED, e a acelerada degradação ambiental a que estão submetidos os ambientes naturais pantaneiros, recomenda-se que as áreas desta formação abrigadas em Unidades de Conservação como a RPPN SESC Pantanal passem a ser importantes sítios para estudos futuros de monitoramento da manutenção de sua diversidade, bem como da resposta destes ambientes à atual condição de alteração climática. Adicionalmente, estes locais devem servir de base para a proposição de planos de manejo sustentáveis para a exploração racional dos produtos não madeiráveis, potencialmente fornecidos pela Attalea phalerata.

\section{CONCLUSÕES}

A área estudada foi identificada como bastante peculiar em relação a outros locais representativos de FESD e FED, especialmente pela presença massiva de Attalea phalerata e ausência de representantes de Myrtaceae. A grande representatividade de espécies pioneiras, em associação à elevada densidade de indivíduos 
dentro desta categoria sucessional, reforça o caráter de formação transicional da Mata com Acuri. Em função da alta porcentagem de espécies decíduas e semidecíduas registradas para a área de estudo, classificou-se esta como sendo representativa de Floresta Estacional Decidual.

\section{AGRADECIMENTOS}

A Luciana L.C. Schwarzbach e Solange R. Zaniollo pelo auxílio na coleta de dados em campo e identificação botânica, respectivamente. Ao SESC pelo apoio financeiro para realização da pesquisa. Ao SESC-Pantanal e RPPN SESC Pantanal pelo apoio logístico e infraestrutura disponibilizados durante a pesquisa de campo. Ao CNPq pela Bolsa de Pesquisa. Aos dois revisores ad-hoc pela cuidadosa revisão e valiosos comentários e sugestões.

\section{REFERÊNCIAS BIBLIOGRÁFICAS}

ARRUDA, L.; DANIEL, O. Florística e diversidade em um fragmento de Floresta Estacional Semidecidual em Dourados, MS. Floresta, Curitiba, v. 37, n. 2, p. 189-199, 2007.

BEIRIGO, R. M. et al. Solos da Reserva Particular do Patrimônio Natural SESC Pantanal. Rio de Janeiro: SESC, 2011.75 p.

BRASIL. Ministério das Minas e Energia. Secretaria Geral. Projeto RADAMBRASIL: Folha SF. 21 (Campo Grande). Rio de Janeiro, 1982. 412 p. (Levantamento de Recursos Naturais, v. 28).

CABRERA, A. L.; WILLINK, A. Biogeografia de América Latina. Washington: OEA, 1980. 122p.

CAMPOS, J. B. et al. Structure, composition and spatial distribution of tree species in a remnant of the Semideciduous Seasonal Alluvial Forest of the Upper Paraná River Floodplain. Brazilian Archives of Biology and Technology, Curitiba, v. 43, n. 2, p. 185-194, 2000.

CAVASSANI, A. T. Floresta Estacional Semidecidual da bacia do médio rio Ivaí - PR: um estudo da dinâmica de regeneração e do potencial uso das espécies na restauração de ecossistemas. 2007. 67 f. Dissertação (Mestrado em Ecologia e Conservação) - Universidade Federal do Paraná, Curitiba, 2007.

CORDEIRO, J. L. P. Estrutura e heterogeneidade da paisagem de uma unidade de conservação no nordeste do Pantanal (RPPN SESC Pantanal), Mato Grosso, Brasil: efeitos sobre a distribuição e densidade de antas (Tapirus terrestris) e de cervos- do-pantanal (Blastocerus dichotomus). 2004. 202 f. Tese (Doutorado em Ecologia) - Universidade Federal do Rio Grande do Sul, Porto Alegre, 2004. CUNHA, C. N.; JUNK, W. J. Year-to-year changes in water level drive the invasion of Vochysia divergens in Pantanal grasslands. Applied Vegetation Science, v. 7, p. 103-110, 2004.

DAMASCENO-JUNIOR, G. A. et al. Florestas estacionais do Pantanal, considerações florísticas e subsídios para conservação. Geografia, Rio Claro, v. 34, n. 143, p. 697-707, 2009.

DISLICH, R. et al. Análise da estrutura de fragmentos florestais do Planalto Paulistano, SP. Revista Brasileira de Botânica, São Paulo, v. 24, n. 3, p. 321-332, 2001.

DUARTE, T. G. Florística, fitossociologia e relações solo-vegetação em floresta estacional decidual em Barão de Melgaço, Pantanal de Mato Grosso. 2007. 162 f. Dissertação (Mestrado em Botânica) Universidade Federal de Viçosa, Viçosa, 2007.

FORZA, R. C. et al. Lista de Espécies da Flora do Brasil. 2010. Disponível em: $<$ http://floradobrasil. jbrj.gov.br/2010/>. Acesso em: 1 maio 2011.

GUARIM NETO, G. Biodiversidade do ecossistema pantaneiro: a vegetação do Pantanal. In: CONGRESSO NACIONAL SOBRE ESSÊNCIAS NATIVAS, 2., São Paulo. Anais... São Paulo: 1992. p. 106-110.

HAMILTON, S. K. et al. Inundation patterns in the Pantanal wetland of South America determined from passive microwave remote sensing. Archiv fur Hydrobiologie, v. 137, n. 1, p. 1-23, 1996.

HOFMANN, G. S. et al. Microclima e a estrutura de formações vegetais. In: SESC. O clima na Reserva Particular de Patrimônio Natural SESC Pantanal. Rio de Janeiro: SESC, Departamento Nacional, 2010. p.11-53.

IBGE. Manual técnico da vegetação brasileira. Rio de Janeiro: IBGE, 1992. 92p.

IVANAUSKAS, N. M. et al. Fitossociologia de um trecho de Floresta Estacional Semidecidual em Itatinga, São Paulo, Brasil. Scientia Forestalis, Piracicaba, n. 56, p. 83-99, 1999.

LIMA JUNIOR, G. A. et al. Distribuição espacial da população de Attalea phalerata (Mart. ex. Spreng), e a relação com o solo em uma Floresta Estacional Decidual no pantanal de Barão de Melgaço, MT, Brasil In: CONGRESSO DE ECOLOGIA DO BRASIL, 8., 2007, Caxambu. Anais ... Caxambu, 2007. p. 1-2.

LOPES, S. F. et al. Caracterização ecológica e distribuição diamétrica da vegetação arbórea em um 
remanescente de Floresta Estacional Semidecidual, na Fazenda Experimental do Glória, Uberlândia, MG. Bioscience Journal, Uberlândia, v. 27, n. 2, p. 322-335, 2011.

LORENZI, H. Árvores brasileiras: manual de identificação e cultivo de plantas arbóreas nativas do Brasil. Nova Odessa: Plantarum, 1992. 352p.

MAGURRAN, E. A. Ecological diversity and its measurement. Princeton: Princeton University Press, 1987. 192p.

MEIRA-NETO, J. A. A.; MARTINS, F. R. composição florística de uma Floresta Estacional Semidecidual Montana no município de ViçosaMG. Árvore, Viçosa, v. 26, n. 4, p. 437-446, 2002. MIRANDA, I. P. et al. Frutos de palmeiras da Amazônia. Manaus: MCT INPA, 2001. 120p. MISSOURI BOTANICAL GARDEN. Tropicos. 2011. Disponível em <http://www.tropicos.org> Acesso em: 1 maio, 2011.

MUELLER-DOMBOIS, D.; ELLENBERG, $\mathrm{H}$. Aims and methods of vegetation ecology. New York: Wiley, 1974. 546 p.

NASCIMENTO, V.L.A. et al. Utilização de frutos de acuri (Attalea phalerata Mart. ex Spreng) por cutias (Dasyprocta azarae) no Pantanal da Nhecolândia. In: SIMPÓSIO SOBRE RECURSOS NATURAIS E SÓCIO-ECONÔMICOS DO PANTANAL, 4., 2004, Corumbá. Anais... Corumbá: Embrapa Pantanal, 2004. (CD-ROM).

NEGRELLE, R. R. B. Composição e estrutura do componente arbóreo de remanescente de Floresta Estacional Semidecidual Aluvial no Pantanal MatoGrossense, Brasil. Revista Árvore, Viçosa, v. 37, n. 6, p. 989-999, 2013.

NIMER, E. Clima. In: Geografia do Brasil: Região Centro-Oeste. Rio de Janeiro: IBGE, 1989. v.1, p. 23-34.

NUNES DA CUNHA, C.; UNK, W. J., Distribution of woody plant communities along the floodplain gradient in the Pantanal of Poconé, Mato Grosso,
Brazil. International Journal of Ecology and Environmental Sciences, New Delhi, v. 27, n. 2, p. 63-70, 2001.

POTT, A.; POTT, V. J. Plantas do Pantanal. Corumbá: Embrapa- CPAP, 1994.

POTT, A. et al. Plant diversity of the Pantanal wetland. Brazilian Journal of Biology, São Carlos, v. 71, n. 1, suppl. 1, p. 265-273, 2011.

ROMAGNOLO, M. B.; SOUZA, M. C. Análise florística e estrutural de florestas ripárias do Alto rio Paraná, Taquaruçu, MS. Acta Botanica Brasilica, São Paulo, v. 14, n. 2, p. 163-174, 2000.

SALIS, S. M. Equação para estimar biomassa da Palmeira Acuri (Attalea phalerata) no Pantanal. Embrapa Pantanal Comunicado Técnico, v. 63, p. 1-4, 2007.

et al. Fenologia de Scheelea phalerata no Pantanal da Nhecolândia, Corumbá, Mato Grosso do Sul. In: SIMPÓSIO SOBRE RECURSOS NATURAIS E SÓCIO ECONÔMICOS DO PANTANAL, 2.,1996, Corumbá. Anais... Corumbá: EMBRAPA-CPAP, 1999.

et al. Fitossociologia de remanescentes de floresta estacional decidual em Corumbá, Estado do Mato Grosso do Sul, Brasil. Revista Brasileira de Botânica, São Paulo, v. 27, n. 4, p. 671-684, 2004.

SANTOS, G. B. et al. Artrópodos associados à copa de Attalea phalerata Mart. (Arecaceae), na região do Pantanal de Poconé, Mato Grosso, Brasil. Revista Brasileira de Entomologia, Curitiba, v. 47, n. 2, p. 2214-224, 2003.

SESC. O clima na Reserva Particular de Patrimônio Natural SESC Pantanal. Rio de Janeiro: SESC, Departamento Nacional, 2010. 84 p.

TERBORGH, J. Keystone plant resources in the tropical forest. In: SOULÉ, M. E. (Ed.). Conservation Biology: the science of scarcity and diversity. Sunderland: Sinauer, 1986. p. 33-44. 